Konrad-Zuse-Zentrum für Informationstechnik Berlin

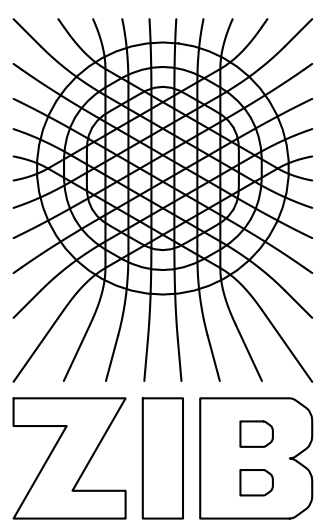

\title{
Planning Problems in Public Transit
}




\title{
Planning Problems in Public Transit*
}

\author{
Ralf Borndörfer ${ }^{\ddagger * *} \quad$ Martin Grötschel** $\quad$ Ulrich Jaeger ${ }^{\ddagger \ddagger}$
}

March 2009

\section{Executive Summary}

Every day, millions of people are transported by buses, trains, and airplanes in Germany. Public transit (PT) is of major importance for the quality of life of individuals as well as the productivity of entire regions. Quality and efficiency of PT systems depend on the political framework (state-run, market oriented) and the suitability of the infrastructure (railway tracks, airport locations), the existing level of service (timetable, flight schedule), the use of adequate technologies (information, control, and booking systems), and the best possible deployment of equipment and resources (energy, vehicles, crews). The decision, planning, and optimization problems arising in this context are often gigantic and "scream" for mathematical support because of their complexity.

This article sketches the state and the relevance of mathematics in planning and operating public transit, describes today's challenges, and suggests a number of innovative actions.

The current contribution of mathematics to public transit is - depending on the transportation mode - of varying depth. Air traffic is already well supported by mathematics. Bus traffic made significant advances in recent years, while rail traffic still bears significant opportunities for improvements. In all areas of public transit, the existing potentials are far from being exhausted.

${ }^{*}$ A German version of this article has been published in M. Grötschel, K. Lucas, and V. Mehrmann, PRODUKTIONSFAKTOR MATHEMATIK - Wie Mathematik Technik und Wirtschaft bewegt, acatech DISKUTIERT, acatech - Deutsche Akademie der Technikwissenschaften and Springer, 2008, pp. 127-153.

${ }^{\ddagger}$ Dres. Löbel, Borndörfer \& Weider GbR (LBW), Salzburger Str. 17, 10825 Berlin, Germany, Email: mailto:borndoerfer@lbw-berlin.de

** Konrad-Zuse-Zentrum für Informationstechnik Berlin (ZIB), Takustr. 7, 14195 BerlinDahlem, Germany, Email: mailto:groetschel@zib.de

${ }^{\ddagger}$ BVO Busverkehr Ostwestfalen GmbH, Am Bahnhof 6, 33602 Bielefeld, Germany, Email: mailto:Ulrich.Jaeger@bvo-bielefeld.de 
For some PT problems, such as vehicle and crew scheduling in bus and air traffic, excellent mathematical tools are not only available, but used in many places. In other areas, such as rolling stock rostering in rail traffic, the performance of the existing mathematical algorithms is not yet sufficient. Some topics are essentially untouched from a mathematical point of view; e.g., there are (except for air traffic) no network design or fare planning models of practical relevance. PT infrastructure construction is essentially devoid of mathematics, even though enormous capital investments are made in this area. These problems lead to questions that can only be tackled by engineers, economists, politicians, and mathematicians in a joint effort.

Among other things, the authors propose to investigate two specific topics, which can be addressed at short notice, are of fundamental importance not only for the area of traffic planning, should lead to a significant improvement in the collaboration of all involved parties, and, if successful, will be of real value for companies and customers:

- discrete optimal control: real-time re-planning of traffic systems in case of disruptions,

- model integration: service design in bus and rail traffic.

Work on these topics in interdisciplinary research projects could be funded by the German ministry of research and education (BMBF), the German ministry of economics (BMWi), or the German science foundation (DFG).

\section{Success Stories}

What good is mathematics in public transit? Three examples elucidate the benefits of mathematics for the customer, the planner, and the stakeholder.

\begin{tabular}{|c|c|c|c|c|c|c|}
\hline Details & Datum & $\begin{array}{l}\text { Abfahrt } \\
\mathbb{G} \text { früher }\end{array}$ & Ankunft & Dauer & Umst. & Verkehrsmittel \\
\hline$\square$ & 30.07 .08 & $15: 24$ & $16: 25$ & $1: 01$ & 2 & (U) $\mathbf{U} \boldsymbol{A}$ \\
\hline ๑ & 30.07 .08 & $15: 31$ & $16: 35$ & $1: 04$ & 4 & Bบ ఆบ $\mathbf{U} \boldsymbol{S}$ Bบ \\
\hline$\square$ & 30.07 .08 & $15: 44$ & $16: 45$ & $1: 01$ & 2 & Bบ $\mathbf{U} \star$ Bบs \\
\hline
\end{tabular}

Fig. 1: Berlin's "trip info" recommends a route.

Electronic Trip Planners. Thumbing through thick timetables and railway guides in order to determine the best connection in a bus, railway, or 
flight network is a matter of the past. Today, bus companies, railways, and airlines offer electronic trip planners, which provide this information via the Internet or via mobile phones in a comfortable and fast way, always up-to-date, and at no charge. To make this service work, correct and comprehensive data is needed first and foremost. The "intelligence" to utilize this data is provided by mathematics: good methods to compute shortest paths in networks. ${ }^{1}$ Appropriate algorithms for this problem are know since the nineteen-fifties. Their use in customer-friendly systems became a reality because of the rapid progress in information technology in recent years.

Examples for electronic trip planners are the "trip info" ("Fahrinfo") of Berlin's public transport company Berliner Verkehrsbetriebe (http://www.fahrinfo-berlin.de), see Fig. 1, the Hafas system, which is used by the German railway company Deutsche Bahn (http://reiseauskunft. bahn.de), and the flight search of Lufthansa (http://www.lufthansa.de). The basic method to compute shortest paths is Dijkstra's algorithm. This method has undergone many refinements and improvements over the years in order to deal with large networks and complex constraints, see [12].

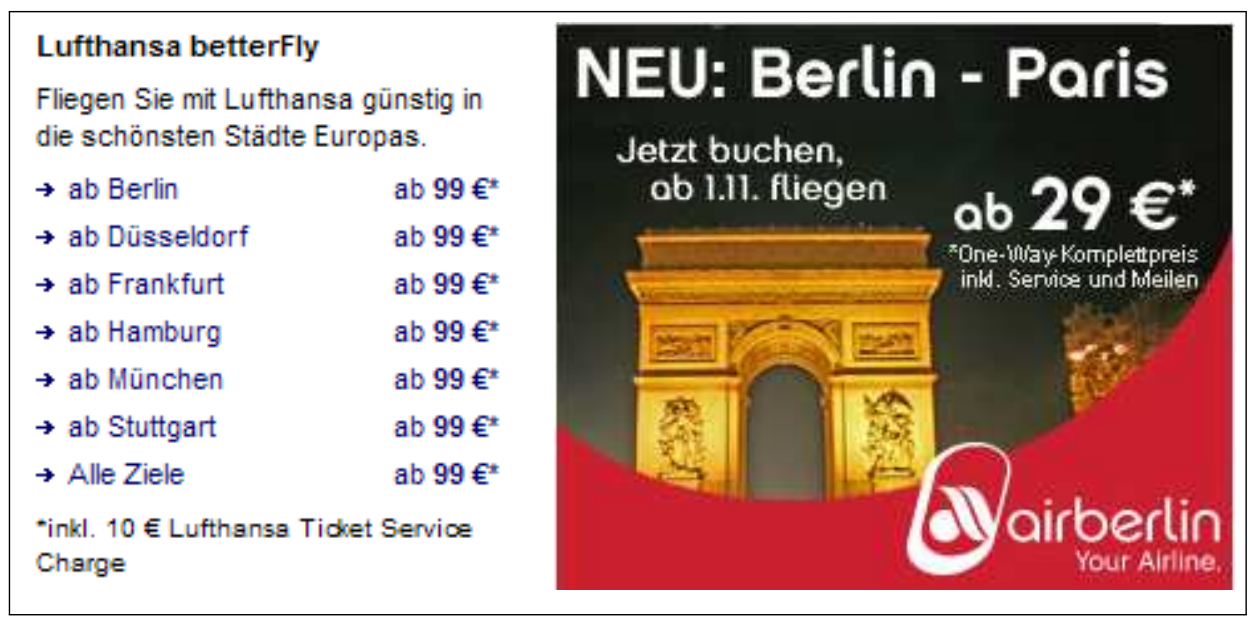

Fig. 2: Flight bargains in the beginning and middle of 2008 (sources: http:// www.lufthansa.com/online/portal/lh/de/specials, 3.1.2008; http:// www. airberlin.com, 3. 6. 2008)

Revenue Management. In the middle of 2008, Lufthansa offered flights to various destinations in Europe for $99 €$, see Fig. 2, Air Berlin promotes flights to Paris as cheap as $29 €$, and sometimes one can find tickets for less than $10 €$. How do such prices come about? They are the result of sophisticated ticket sales strategies known as revenue or yield management. The idea is as follows. Once an airline has published a flight in its schedule,

\footnotetext{
${ }^{1}$ Customers sometimes complain that they can find better or cheaper routes than a trip planner. This is, however, not a mathematical problem, but usually due to parameter settings such as "minimum transfer times".
} 
it is essentially clear what the costs will be. The goal is then to maximize the revenue. There were originally two strategies to do this: the classical carriers charged high prices (and had empty airplanes on certain flights), while the low costs carriers filled their airplanes solely with cheap tickets. Today, all airlines pursue (depending on the company) different mixtures of these strategies. The clou about it is in the permanent adjustment of the booking classes and prices to the demand that has already materialized and the demand that is yet expected. Many airlines use mathematical methods of stochastic optimization to do these adjustments. On the basis of such forecasts, it can be reasonable to sell, at certain points in time, residual capacities at very low prices, such that at least some revenue is generated instead of flying empty seats.

The above described and at present commonly used form of revenue management was developed around 1990. In this context, the famous competition between American Airlines and the low cost carrier PeopleExpress is often mentioned, because AA finally won the fight by introducing "Super Saver" and "Ultimate Super Saver" tickets, which were sold using yield management methods. On the occasion of the bestowal of the INFORMS Edelman Award in 1991, AA provided evidence that revenue management created an additional revenue of 1.4 billion USD in the period from 1988 to 1991 [32]. After additional improvements, a benefit of even 1 billion USD per year was reported [9]. The most popular revenue management method is the EMSR rule (expected marginal seat revenue), which states that one should sell tickets of some booking class for a flight as long as the expected profit is positive [26]. Starting from this basic form, researchers and practitioners have developed a large variety of methods to control ticket sales, ranging from the consideration of individual flights ("leg control"), via the inclusion of simple network effects ("segment control"), to the treatment of entire itineraries ("origin destination control"), see [34] for a recent overview of the state of the art in this area of research in stochastic optimization.

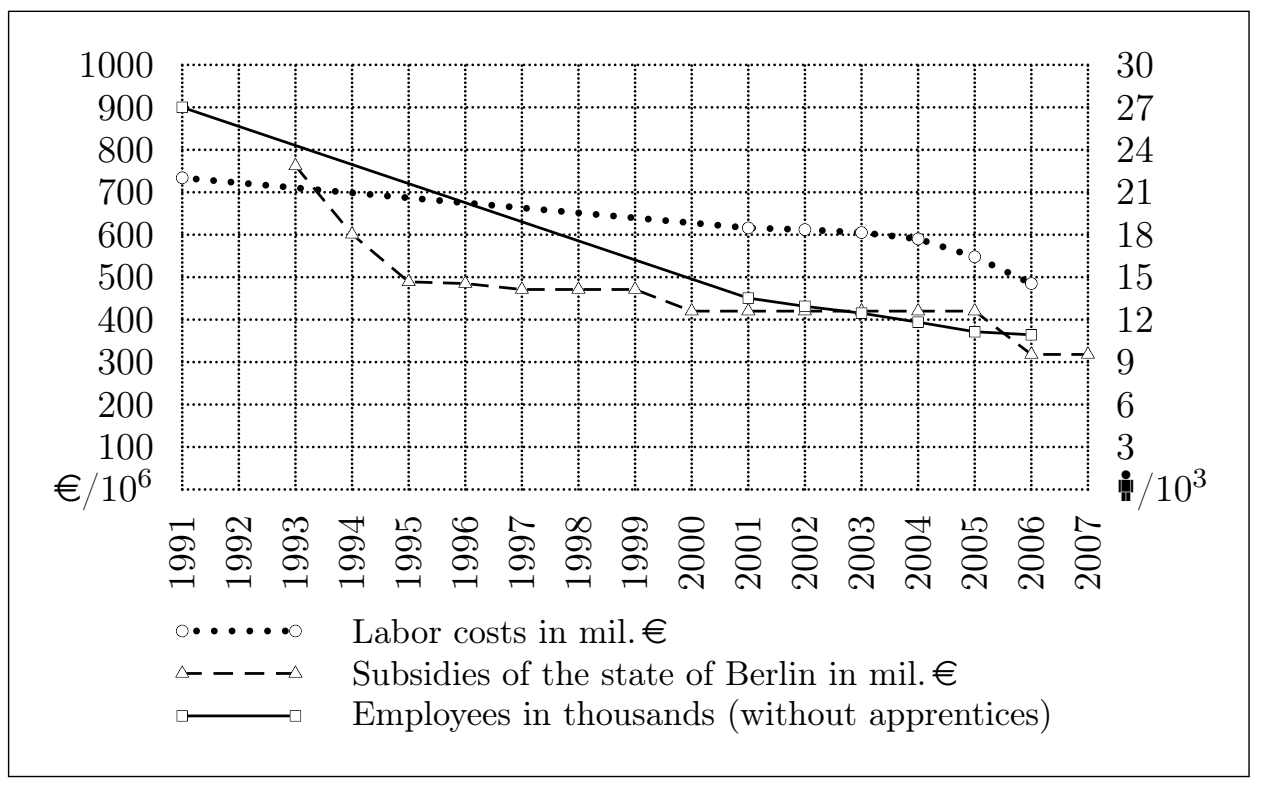

Fig. 3: Berlin's public transit company BVG in numbers (source: [33]) 
Vehicle and Crew Scheduling. Fig. 3 illustrates the development of Berlin's public transit company BVG over recent years by means of three statistics: the number of employees descended from 27002 in 1991 to 10982 in 2006, labor costs fell in the same period of time from 734 mil. $€$ to 485 mil. $€$, and state subsidies sank from 762 mil. $€$ in 1993 to 318 mil. $€$ in 2006 [33]. These remarkable reductions could be achieved without changing the level of service. They are not only, but to a significant extent, results of mathematical optimization. As Andreas Sturmowski, CEO of the BVG, put it [33]: "The use if IT-based planning systems allows for significant improvements in the planning processes of the BVG! Resource allocation is optimized. Increases in the productivity of vehicle and crew utilization [are achieved] by minimizing deadhead trips, [...], better use of depot capacities, $[\ldots$, and $]$ reductions in staff requirements by optimized duty schedules [...]." (translation by the authors). All this is made possible by methods of combinatorial and mixed integer optimization, which can deal with the enormous problem sizes that are typical for this area. This "computing power" leads to both significant speed-ups of and to quality improvement in the planning process. Customers do not directly notice this progress; however, the indirect impact on ticket prices and state subsidies is significant.

Mathematical methods for vehicle and crew scheduling are today sold as standard modules in the market leading planning systems. In the area of public transit, such systems are, among others, MICROBUS 2 of IVU Traffic Technologies AG (http://www.ivu.de) from Berlin, HASTUS of the Canadian company Giro Inc. (http://www.giro.ca), and Turni by the Italian company Double-Click (http://www.turni.it/page001.htm), in air traffic the systems NetLine by Lufthansa Systems GmbH (http://www.Ihsystems.com) and Carmen of the US Jeppesen group(http://www.carmen. se), in rail traffic the system railRMS by Jeppesen or the optimization modules of the US company Innovative Scheduling, Inc. (http://www.innovativescheduling.com). The state of the art of mathematical research is documented by the proceedings of the tri-annual international CASPT and the German Heureka conferences [10, 11, 14, 18, 35, 36].

\section{PT Planning Problems: Survey and Status Quo}

Public transit (PT) is of high relevance for every society. Tab. 1 illustrates at the example of some statistics on Berlin's public transit company (BVG), the German railway company Deutsche Bahn (DB), and the German airline Lufthansa ( $\mathrm{LH}$ ) for the business year 2006, that PT is also an important sector of the economy.

In the future, the importance of public transit will increase because of the surging costs of individual traffic. In Germany, in the summer of 2008, a single tankful of gasoline cost about $75 €$, which is nearly the price of a monthly BVG public transit ticket for the tariff zone AB, which contains the entire area of the state of Berlin (about 40 square kilometers).

Public transit can be subdivided into the following three areas: 


\begin{tabular}{lrrrr}
\hline company & pkm/year & employees & turnover/Euro & profit/Euro \\
\hline BVG & 4,074 bil. & 12.685 & 0,636 bil. & 23 mil. \\
DB & 74,788 bil. & 229.200 & 30,053 bil. & 1.680 mil. \\
LH & 110,330 bil. & 93.541 & 19,849 bil. & 845 mil. \\
\hline
\end{tabular}

Tab. 1: Public transit 2006 in Germany; sources: [4], [15], [16]. The respective figures must be interpreted, because some of them are derived in different ways. The BVG, e.g., reports for the number of employees the "average number of employees over the year", including apprentices. The figures of DB and LH include, among other things, cargo traffic.

- public mass transit, i.e., bus, tram, subway, and commuter trains (BT),

- long and short distance passenger rail traffic (RT),

- civilian passenger air traffic (AT).

We will denote these in the following shortly as bus, rail, and air traffic. The planning problems appearing in these areas can be classified from a business point of view as follows:

- scheduling (e.g., vehicle and crew scheduling)

- control (e.g., delay and disruption management)

- (network) design (e.g., infrastructure, timetable)

- regulation of competition (e.g., tenders).

Mathematical methods are already used in all these fields. However, the maturity of the technology and the penetration of practice is different. In scheduling, mathematical optimization methods are well established as an industry standard, the mathematical control and design of public transit systems is (with the notable exception of air traffic) in a research state, while the mathematics of $\mathrm{PT}$ regulation is still in its infancy. There are also differences depending on the transportation mode. One reason is that the planning problems in bus, rail, and air traffic may be similar, but they are not identical. Technical and organizational conditions as well as the market environment influence the structure of the planning process, as well as the definition and the characteristics of the individual tasks, which in turn has its effects on mathematical solution approaches. At present, the use of mathematics is most advanced in air traffic, and least common in rail traffic.

We survey in the following the state of the most important developments. 


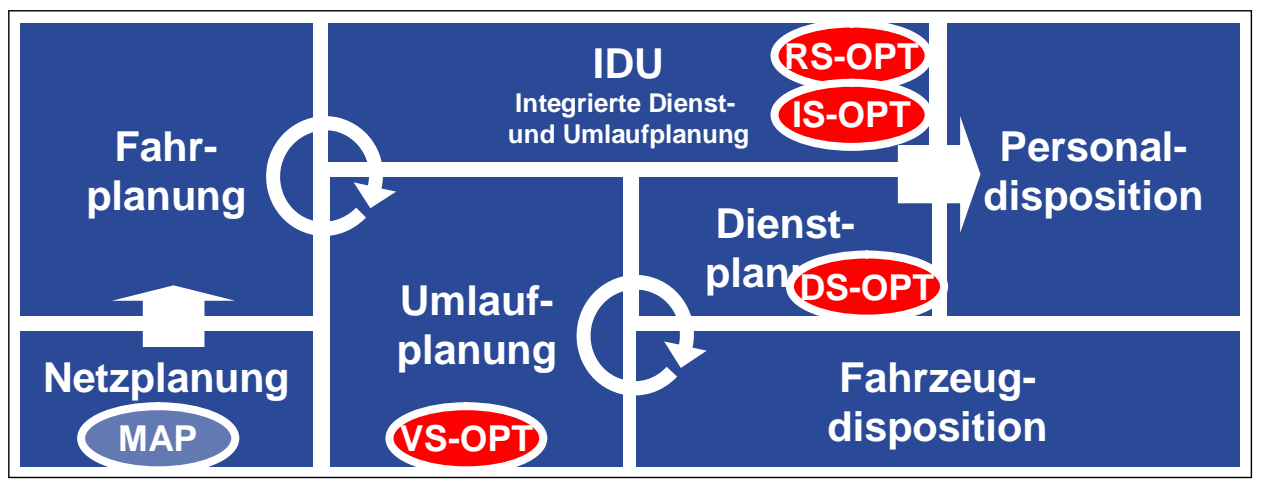

Fig. 4: Bus scheduling with MICROBUS 2 (source [28])

\subsection{Scheduling}

Scheduling determines - on the basis of a fixed timetable - the use of vehicles and crews. Operating a bus (including general overhead) costs approximately $250000 €$ per year in Germany. About $70 \%$ of this amount are crew costs, and $25 \%$ vehicle costs. In rail and air traffic, the vehicle costs take a larger share of the total costs, in particular, because of higher fuel expenses.

The problems to plan vehicles and crews can, in their basic forms, be formulated in terms of standard models of combinatorial optimization. One can view them as multi-commodity flow problems or as path covering problems. These models are well studied and can be solved in dimensions which make a treatment of realistic and relevant scenarios possible at a high level of detail. However, technical constraints, legal prescriptions, and company agreements increase the complexity of the problems to such an extent that specialized methods have to be developed. These have reached a mature state and are today (as already mentioned) offered by software companies all over the world.

The particular structure of the planning process and its subdivision into individual problems depends on the perception of the operation and differs by countries, transportation modes, and software companies. Fig. 4 illustrates the view of the software company IVU Traffic Technologies AG on public transit. The planning system MICROBUS 2 can be used to schedule, on the basis of a given network and timetable, vehicle rotations and crew duties for a day of operation, which are subsequently concatenated to vehicle and duty rosters. These individual steps are supported by optimization tools, which are marked in red. We give in the following a synopsis and explain the problems both from an applied and from a mathematical point of view. 
Vehicle Scheduling. The first successful applications of mathematical methods in public transit dealt with bus scheduling. This planning step is about the construction of sequences of timetabled and deadhead trips, the so-called vehicle rotations, such that every "timetabled trip" (a trip listed in the timetable) is operated by a suitable vehicle. The planning horizon is usually a single day of operation. A bus departs from its depot in the morning and returns in the evening. The objective is to service all timetabled trips and to minimize the corresponding costs (of driving and waiting). This task can be formulated mathematically as a multi-commodity flow problem, see Fig. 5. In the case of Berlin's public transport company BVG, this leads to an optimization problem with 100 million variables, which can be solved by modern algorithms in less than one hour on a standard desktop computer to proven fleet optimality [27]. Using this method, BVG reports savings of 38 buses in a single depot (Spandau) in 2003, i.e., about $20 \%$ of the vehicles, and of 377 hours of unproductive waiting time [30].

In air traffic, vehicle scheduling is usually subdivided into two steps, namely, fleet assignment and tail assignment. Fleet assignment decides which type of aircraft is used to operate a specific flight. In the subsequent tail assignment

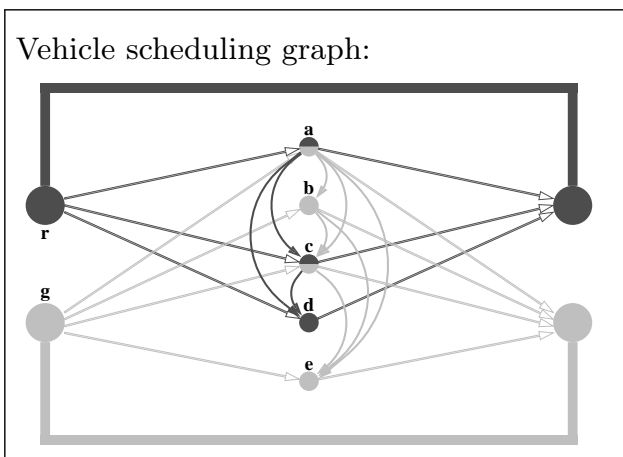

Fig. 5: Vehicle scheduling in public transit (see [27])

The vehicle scheduling problem in public transit can be mathematically modeled as a multi-commodity flow problem. The model is based on a scheduling graph, which connects the timetabled trips by all possible deadhead trips. Vehicles of different types "flow" through this graph in such a way that every timetabled trip is covered by a rotation of a vehicle of a suitable type. Formally, the model can be described as follows. We denote by $\mathcal{D}$ a set of vehicle types, by $\mathcal{T}$ a set of timetabled trips, by $A^{d}$ a set of arcs for all possible deadhead trips for every vehicle type $d$, and by $\kappa^{d}$ the number of available vehicles of type $d$. We introduce a $0 / 1$ variable $x_{i j}^{d}$ for every $\operatorname{arc}(i, j)$ and every vehicle type $d . x_{i j}^{d}$ encodes if a deadhead trip $(i, j)$ is operated by a vehicle of $d$ (in which case $x_{i j}^{d}=1$ ) or $\operatorname{not}\left(x_{i j}^{d}=0\right)$.

The corresponding formulation as an integer linear program is:

$$
\begin{aligned}
\min \sum_{d,(i, j)} c_{i j}^{d} x_{i j}^{d} & \\
\sum_{d,(t, j)} x_{t j}^{d} & =1, \forall t \in \mathcal{T} \\
\sum_{t j}^{d}-\sum_{d,(i, t)}^{d,(t)} x_{i t}^{d} & =0, \forall t \in \mathcal{T}, d \in \mathcal{D}, \\
\sum_{(d, j)} x_{d j}^{d} & \leq \kappa^{d}, \forall d \in \mathcal{D} \\
x & \geq 0 \text { and integer. }
\end{aligned}
$$

Berlin's public transit company BVG distinguishes about 50 different types of vehicles, 28000 timetabled trips, and approximately 100 million deadhead trips. This translates via the above model into a multi-commodity flow problem with about 100 million variables and several hundred thousands of equations and inequalities. 
step, rotations for the individual airplanes of each fleet are constructed, including necessary maintenance activities. There are two reasons for this subdivision. First, operation costs for large and small airplanes differ much more than for buses, such that fleet assignment is much closer related to network design than bus scheduling to bus network design. Second, the planning horizon of the tasks is different (tail assignment is closer to the day of operation). Fleet assignment can be modeled as a multi-commodity flow problem, similar to bus scheduling, while tail assignment leads to path covering problems that are solved using column generation methods, see Fig. 6. Today, optimal vehicle rotations for an entire bus company or airline are computed routinely.

Vehicle scheduling in rail traffic features additional technical constraints, which depend, among other things, on the infrastructure at railway and shunting stations and on the driving dynamics of individual trains. Such constraints increase the complexity of the associated models substantially. At present, the mathematical methodology is not good enough to deal with problems of relevant sizes. Vehicle scheduling in rail traffic is currently to a wide extent still a matter of manual planning and heuristic methods.

Crew Scheduling. Crew scheduling is commonly subdivided into two consecutive steps: crew/duty scheduling and crew/duty rostering. Crew scheduling is about the construction of a number of crew rotations, the so-called duties (in bus traffic) or pairings (in air traffic), which are not yet assigned to specific crews; this step accounts for legal regulations and aims at cost minimization. Crew rostering subsequently concatenates anonymous duties to longer rosters over some planning horizon and assigns them to concrete persons; here, fairness considerations and employee preferences are taken into account.

Airline crew scheduling is the area where the first big successes in mathematical crew scheduling were achieved. Labor costs of large airlines range in the billions and are, next to fuel costs, the second largest individual cost factor [1]. At the beginning of the nineteen-nineties, it became possible for the first time to solve large crew scheduling problems with thousands of flights to proven optimality [21]. Since then, optimization technology has made rapid progress and is now established as an industry standard. Today, all major airlines use mathematical "crew optimizers" [37]. Methodologically, these systems are based on set partitioning models, which can deal with the complex rules for breaks, rest periods, etc. These models are solved by column generation methods, see Fig. 6.

These methods have been transfered with some delay to bus and in some places also to rail traffic. However, the problems in these areas have special 
combinatorics of their own, which, among other things, are caused by different degrees of freedom for crew reliefs (e.g., in bus traffic one changes crews "on the fly", i.e., during a trip, which is obviously impossible in airline crew scheduling); on the other hand, duty schedules in bus traffic are constructed for a single day of operation, while airline crew scheduling is typically done for a period of several weeks or a month.

Summarizing, crew scheduling is the area in which the use of mathematical methods in public transit is most advanced. Occasionally this is criticized, as documented, e.g., by the headline of the Atlanta Constitution of August 13, 1994: "Delta to furlough 101 more pilots in bid to cut costs". But such criticism has no bearing on the mathematical methodology. Indeed, it is no

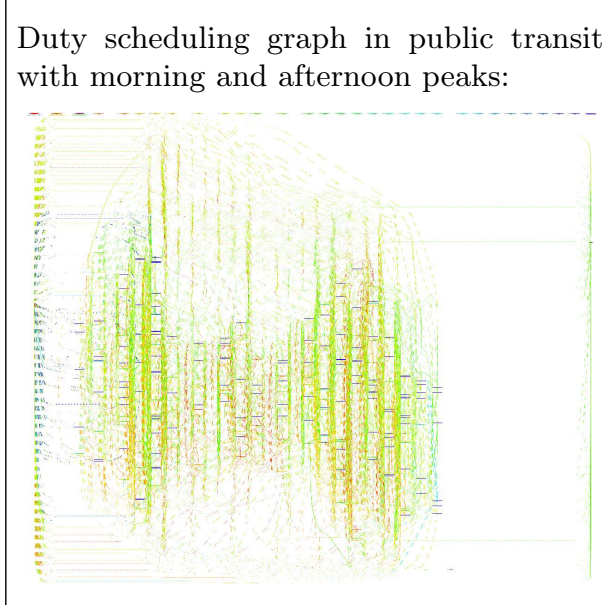

Fig. 6: Duty scheduling in public transit[6]

The duty scheduling problem in public transit can mathematically be understood as a path covering problem. Similar to vehicle scheduling, one constructs a scheduling graph, in which indivisible units of (driving) work, the so-called duty elements, are linked by all possible connections. Driver duties correspond to "paths" in this scheduling graph, and every duty element has to be covered by such a duty. The difference to vehicle scheduling is that complex rules for the feasibility of duties such as break rules have to be observed, which depend on the form of the duty in its entirety and which can not be decided locally. Consequently, a mathematical duty construction can no longer efficiently be done in terms of variables for individual concatenations of duty elements, but must introduce variables for entire duties, which makes these models larger and more difficult to solve.

Following this line of thought leads to a formally very simple model, a so-called set partitioning problem. It considers for some set of duty elements $I$ the set of all possible duties $J$. For every such duty $j$, a $0 / 1$ variable $x_{j}$ is introduced. It indicates whether duty $j$ is included in the duty schedule (in which case $x_{j}=1$ ) or not $\left(x_{j}=0\right)$ ). For every duty element, a constraint stipulates that exactly one duty covering this element must be chosen. For large, industrial problems it is not possible to handle this formulation explicitly, because there can easily be billions of duties even for small problems with only 200 trips (duty elements) [22]. However, such models can be solved implicitly using so-called column generation methods, which generate (in a mathematically precise sense) at any time the potentially interesting duties in a dynamic way $[3,13]$.

The corresponding formulation as an integer linear program is

$$
\begin{aligned}
\min & \sum_{j \in J} c_{j} x_{j} \\
& \sum_{j \ni i} x_{j}=1, \quad \forall i \in I \\
& x \geqslant 0 \text { and integer. }
\end{aligned}
$$

Nowadays duty scheduling problems in bus and air traffic with several hundred or even thousands of crews are solved on a routine basis $[2,6]$. 
problem to optimize for social criteria. Managers, however, usually focus on cost reductions.

\subsection{Control}

Fig. 7 illustrates that controlling the implementation of a schedule is as important as constructing it. Bad weather, accidents, technical damages, and strikes can cause disruptions. For example, the Chicago Tribune reports on December 27, 2007: "According to its pilots union ..., United has run low on crews to fly its planes. That's a result of lean staffing, scheduling practices and freakishly bad weather that have caused large numbers of pilots to hit the maximum number of monthly duty hours allowed by federal regulators well before December's end." There are different possibilities to face such difficulties.

Stochastic and Robust Optimization. A theoretically satisfactory possibility is to use methods of stochastic optimization, that try to anticipate disruptions in a probabilistic way. A main obstacle to the application of stochastic methods, however, is the limited availability of expected values and probability distributions in practice. For this reason, a simplified variant of stochastic optimization, the so-called robust optimization approach, has been investigated in recent years. It only accounts for certain uncertainty intervals in the data by means of a worst case analysis. This method has not made it into practice yet. Real-world planning systems take some precaution by introducing buffers at critical points.

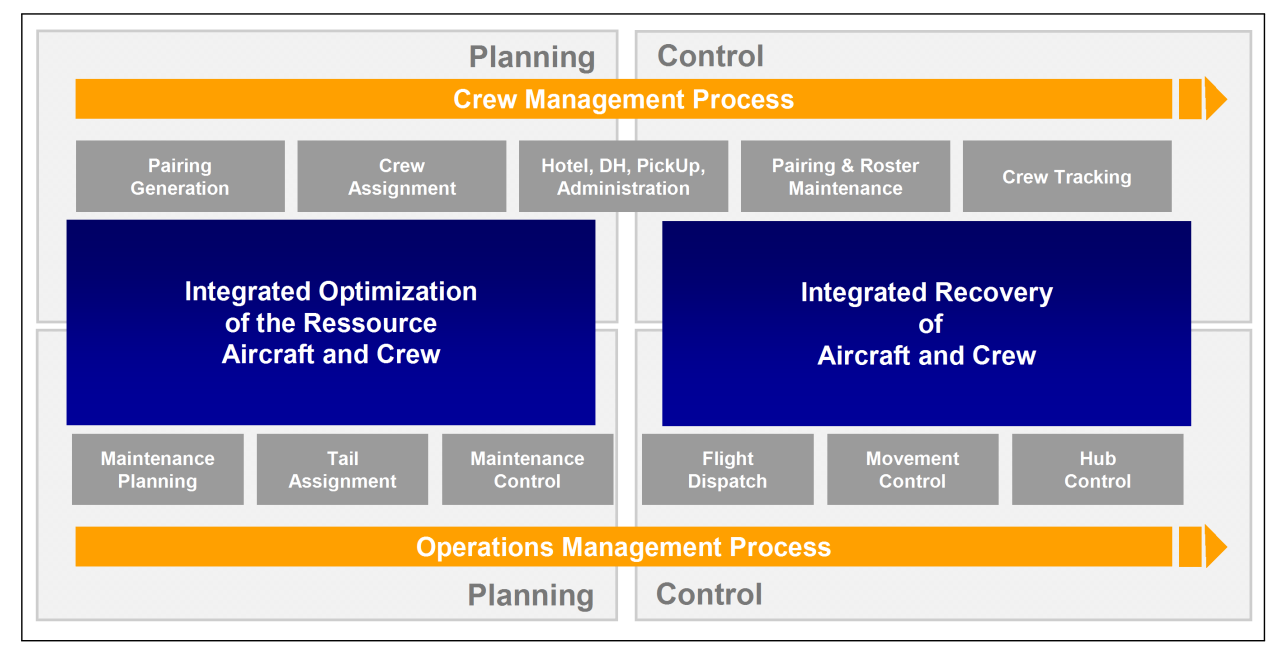

Fig. 7: Airline planning and control with NetLine (source [8]) 
Online Optimization. Stochastic and robust optimization pave the way to overcome disruptions by instantaneous re-planning. Re-planning itself is a topic of online resp. real-time optimization, a method that searches for recovery operations that lead from a disrupted state back to a stable state as fast as possible and, in this way, reduce the damages caused by disruptions as much as possible. Online optimization methods are, e.g., currently successfully used for dispatching the "yellow angel" service vehicles of the German automobile club ADAC [20]. In principle, bus and rail traffic also have the necessary IT infrastructure at their disposal. However, in these areas disruption management is usually done by experienced dispatchers. In air traffic, first successes with mathematical methods are reported, e.g., at the American carrier Continental Airlines [38]. The software companies have started to take up the topic.

Inadequate control leads not only to customer dissatisfaction, but also to enormous costs. For example, airlines must pay passenger overnights and arrange ferry and extra flights, bus and railway companies do not get their full payment in case of quality impairments.

Robust and real-time optimization are new and topical branches of research, which can only have an impact if a close cooperation with practice can be established.

\subsection{Service Design}

Service design can be subdivided into three important areas: infrastructure construction, vehicle procurement, and line, timetable, and fare planning.

Infrastructure Construction. The arguably most important topic of planning in public transit are decisions about investments in infrastructure such as the construction of airports, railway tracks, railway stations, or bus depots. Infrastructure investment costs are huge. The new ICE (intercity express $=$ high speed train) line from Cologne to Frankfurt, e.g., cost more than 5 bil. $€$, the construction of the new Berlin airport BBI is estimated at $2-5$ bil. $€$. Once taken, decisions about infrastructure constructions can only be changed over a very long period of time. It is therefore necessary to conduct an extremely careful analysis of all possible alternatives.

The decision methodology that is currently used in infrastructure construction is based on the simulation of a few scenarios in the sense of case studies (usually accompanied by a super-charged political discussion). This method is not adequate to deal with projects that interact in a complex way with the entire transportation infrastructure. In rail traffic, to mention one example, there is currently a discussion in Germany whether the construction of new, 
expensive high speed tracks produces the biggest benefit or if a bundle of many small investments would be more useful. Infrastructure investments in bus traffic focus on the selection of sites for bus depots, a topic that is currently particularly important because of merger activities. Here, network optimization computations are necessary. To the best of our knowledge, however, this is not done to an extent that would be worth mentioning. From a mathematical point of view, problems of similar structure come up in telecommunications. The German research network association (DFNVerein), an organization that operates the research Internet in Germany, e.g., has undertaken a simultaneous site and network optimization for the German science network (currently X-WIN) on a regular basis for many years [5]. The methods that have been developed in this context could be a starting point for mathematical approaches to infrastructure design in public transit.

Vehicle Procurement. The procurement of airplanes, trains, buses, etc. constitutes a major cost factor in public transit. An A380 passenger plane costs more than 200 mil. $€$, an ICE3 high speed train more than 20 mil. $€$, a $25 \mathrm{~m}$ articulated bus $500000 €$. The German railway company Deutsche Bahn operates 236 ICE-train units [15], Berlin's public transport company BVG more than 1,300 buses [4], the German airline Lufthansa 411 passenger planes of various types [16]. The assessment of the fleet size that is necessary to operate a flight, rail, or bus network is sometimes still done using simple rules of thumb [24, 31], such as

$$
\left\lceil\frac{\text { length of line } \times \text { frequency }}{\text { average speed }}\right\rceil+\text { reserve; }
$$

sometimes, instruments of operational scheduling are also employed. Usually, decisions are taken as a compromise on the political level, on which mathematical considerations do not always play an important role.

Line, Timetable, and Fare Planning. Line planning and timetabling deal with the specification of subway, tram, and bus line routes, travel frequencies, and the concrete departure and arrival times of the vehicles. These two planning steps belong arguably together, at present, however, they are carried out sequentially, mainly because the mathematical methods for a simultaneous treatment are not available. For both problems, research codes exist. In line planning, one can deal with medium sized instances, see, e.g., [7]. Somewhat more advanced is periodic timetable optimization, which was used, e.g., to compute the timetable of the Berlin subway [25]. All these approaches act on the assumption of a given, fixed demand. 
Fare planning investigates how the demand for public transit changes in response to a change in ticket prices. To this purpose, one must model user demand patterns and, in this way, forecast the demand subject to varying prices. Very simple demand models are based on price elasticities. The problem with these models is that elasticities have only been empirically estimated in isolated places (at certain times and places) and with rather different results. Constant price elasticities can not be used to predict substitution effects. More modern models, based on discrete choice approaches, have been developed in the meantime and can now be solved to a moderate extent [29], but they have not been applied in practice. Air traffic is an exception. Here the already discussed revenue management methods are used with great success.

The long term goal must be to deal with line, timetable, and fare planning in an integrated way, because all these problems are intimately related to each other. However, as the individual problems are not completely mastered yet, we are still far away from an optimization at a system-wide level.

\subsection{Regulation}

Service design, as discussed in the previous section, is based on the current regulatory framework. All over the world, there is a discussion how far the public transit market can be deregulated and in what way competition can be introduced into this system. The deregulation in air traffic has led to a veritable boom. There was an enormous reduction in ticket prices and a surge in passenger volume. Similar effects are hoped for rail and bus traffic, however, it is not clear what the right deregulation recipes are. Hitherto experiences in different parts of the world produce a mixed picture. The deregulation of the railway sector in Great Britain was hailed as a major breakthrough in the beginning, then led to a disaster, and now, after changes in the general conditions, a success is beginning to show. Another example is the redesign of the bus transport network in Santiago de Chile, which took place in spring 2007. It resulted in a complete breakdown of the metropolitan traffic that nearly forced the government of Chile to resign. The mathematical analysis of regulatory measures can help to identify and prevent undesirable consequences of changes in the design of a traffic system.

An example of how this could look like in practice is given by the German "railway infrastructure usage regulation" ("Eisenbahn-Infrastruktur-Benutzungsverordnung", EIBV), that is supposed to set the rules for a competitive access to the German railway network. The EIBV states in $\S 9$ para. 5: "In the decision process ... the operator of the railway system must compare the fees for the controversial slots and ... in case of a conflict between more than two slots he must give precedence to those slots whose sum of 
fees is collectively maximal." (translation by the authors). This is an optimization decree. It obliges the German railway network operator DB Netz AG to solve a track allocation problem, and the German network regulation agency Bundesnetzagentur to make sure that an optimal solution is determined. Without mathematical optimization, a non-discriminatory access in the sense of this regulation is not conceivable. This is "overlooked" by all involved parties, usually with the justification that negotiated solutions are better suited. Basically very similar problems arise in airport slot allocation in air traffic and in the tendering of bus lines. These topics lead from a mathematical point of view to questions in combinatorial auction theory and in algorithmic game theory, which are at present debated in the economic research community and for which several Nobel prizes in economics, the last one in 2007, have been awarded.

There are by now successful applications of this methodology in the tendering of transportation contracts in logistics [23] and firms have emerged which market the corresponding auctioning platforms (combine.net). In public transport, however, such methods have not been applied yet.

\section{Strengths, Weaknesses, and Challenges}

Section 3 described planning problems in public transit from an operator's point of view. This section offers a synopsis from a mathematical perspective, elucidates strengths and weaknesses, and describes some of the current challenges.

\subsection{General Conditions for the Use of Mathematics}

Centralized Forms of Organization. The public transport modes bus, rail, and air traffic are characterized by relatively centralized forms of organization. Except for the area of operations control, there is sufficient lead time for all planning problems, such that it is possible to compute alternatives and variants. These are in principle excellent premises for the use of mathematical planning methods.

Availability of Data and Information Technology. In order to use optimization methods, detailed and precise data is required. Progress in information technology makes data available in ever larger quantity and in ever increasing quality. Radio data transmission, GPS, and electronic ticketing systems are abundant sources of information about user behavior and the state of operation of a public transit company. Far-sighted planning, 
however, needs considerable amounts of additional data. Vehicle scheduling, e.g., requires thousands of lengths of alternative deadhead trips, which can be used potentially. These so-called degrees of freedom are not automatically available, at least not in sufficient quality, and their procurement is often a major effort during the introduction of optimization systems. In some areas obtaining the necessary data is difficult in principle. In fare and service design in public transport it would actually be necessary to forecast user behavior in the presence of changes of prices or changes of the service level. Here, one usually uses statistical origin-destination matrices. The occasionally used price elasticities are based on fairly unreliable data. In these areas, a trustworthy data basis must be created in order to develop accurate methods of service design.

Public transit as well as the associated planning methods benefit hugely from the breathtaking developments in information technology. Cellular phones, radio data transmission, etc. made the use of quantitative, mathematical planning methods possible.

Complexity. Small public transit companies may very well produce good results using manual planning under constant general conditions. But even there, quality and efficiency requirements are increasing. For large public transit companies with thousands of buses, tens of thousands of employees, and countless connections, planning by "peering", by analyzing Excel sheets, or by arguing in terms of individual aspects is neither the state of the art nor best practice. The same holds for expensive investments in infrastructure constructions, which change the logistics of entire regions. It is again and again striking to see that airports are constructed without connections to the subway or commuter train system. Such mistakes are due to the lack of a "global point of view" and the fact that network effects of individual decisions are not understood. Infrastructure policy decisions should actually be embedded in comprehensive network models, that describe the long term transportation demands of a region, and that, in particular, take inter-modal effects into account. Planning activities of this type are currently primarily done by engineering companies from the construction industry, that know all about the technical aspects, but that do not command the mathematical methods that are necessary to master the complex dependencies. Furthermore, planning is complicated by government regulations, company agreements, and other rules and standards, which are not written with an eye on the consequences that an increase in complexity incurs on the subsequent planning process, see Fig. 8 for a typical example.

Public transit planning problems are highly complex. Mathematics can not eliminate complexity, but it offers methods to cope with it. Indeed one can prove that the planning problems that come up in public transit are in a 


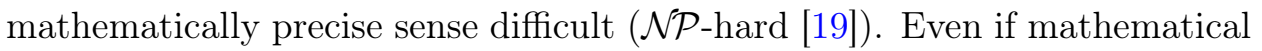
methods may not necessarily be able to produce provably optimal solutions, they can often compute the deviation from optimality or similar quality guarantees, something that no other methodology provides. In general parlance: Nothing works without mathematics.

It is alarming that this insight is not yet realized by all public transit companies. In many cases manual planning is still the method of choice, even in large enterprises. Really astounding, however, is that even some engineering consultancies and software developers in this area argue that optimization would only deal with special aspects, while manual planning would consider the problem in its entirety - a completely absurd claim from our point of view. Actually, the real goal must be to master the complexity of PT systems by combining traffic engineering know-how with the mathematical methodology. This is the only way to face the grand challenges in integrated planning and in the design of entire transportation systems. Investments in mathematical decision methods are marginal in comparison to investments in infrastructure construction, vehicles, and crews, the effects, however, that one can achieve in this way, are considerable.

Standardization. Many public transit companies tend to view themselves as unique and believe that the problems arising in their case are so special, that they need particular attention. In addition, the wish list that planners, managers, and work council members present to software companies is virtually unlimited. This type of atomization of problems is a major obstacle to the further development of the planning methodology. Only if planning problems are standardized and if data is available in standard formats, one can start a serious and sustainable investigation of the underlying mathematical problems over a longer period of time, analyze their mathematical structure, and develop special purpose methods, such that one can hope for practical solutions even for the problem dimensions in public transit. Areas that succeeded in standardization have enjoyed the development of powerful methods over time. The question about the "correct" definition of the individual planning problems is often controversial. However, the benefits of standardization are usually so large that it is better for a company to attune its processes, than to work with tailor-made individual solutions, which do not evolve at the the same pace.

As usual, standardization is most advanced in air traffic. Here, the planning process is subdivided into generally accepted subproblems, for which data interfaces and powerful optimization methods are available. By now, bus traffic has established similar standards in the area of operational planning. In service design, standardization sometimes evolves as a result of the dominating market position of individual suppliers, e.g., by means of ptv's 
$\S 8$ Flight times of crew members

(1) The unrestricted flight time of every crew member between two rest periods is 10 hours. It is feasible to extend the flight time according to sentence 1 up to 4 times for up to 4 hours a time in 7 consecutive days; here, the sum of the extensions must not exceed 8 hours in any 7 consecutive days. The period of 7 consecutive days starts at any one time at 00.00 Greenwich Mean Time (GMT) of the first day and ends at 24.00 GMT of the seventh day. For a pilot, who acts in his flight time according to sentence 1 as an aviator all or in parts without support by another crew member, paragraphs 2 and 3 do not apply.

(2) The maximum flight time extension for crew members of 4 hours according to paragraph 1 is diminished

1. by 1 hour, if the flight time is longer than 2 , but less than 4 hours,

2. by 2 hours, if the flight time has an intersection of 4 or more hours with the time window between 01.00 and 07.00 local time of the departure airport (winter time).

(3) A diminished flight time extension according to paragraph 2 is further diminished

1. by another hour, in case of more than 3 , but less than 6 landings,

2. by another 2 hours, in case of more than 5 landings.

(4) If the aircraft crew is larger than the mandatory minimum, and if suitable sleeping accommodation is available on board in a compartment that is separated from the cockpit and the cabin, the regulation authority can accept a request in writing to extend the flight time according to paragraph 1, sentence 1, of up to 8 hours for two times within 7 consecutive days. Here, the time that a crew members spends in aviation and operation of the aircraft must not exceed 12 hours.

Fig. 8: From the 2nd executive order on work rules for aircraft crews (2. Durchführungsverordnung zur Betriebsordnung für Luftfahrtgerät (2. DVLuftBO), translation by the authors)

product VISUM. Such quasi-standards are in principle a possible basis for the development of optimization methods, but they would have to be extended, because they were primarily designed for simulation, and not for optimization, purposes. In rail traffic, on the other hand, nearly every company pursues an individual approach, such that results are very difficult to transfer from one railway company to another. First efforts on standardization are also being made, e.g., in the OpenTrack project of ETH Zurich.

Regulation and Deregulation. It is general consensus that public transport is a matter of public interest. However, it is controversial how far the public interest reaches and how the service is organized, i.e., how the state is supposed to get to the desired welfare maximum for its citizens. In the past it was believed that the citizens are best off if state-run, monopolistic operators run the public transportation system. This concept got under dispute. The European Union pursues a clear deregulation policy in public transport 
as well as in other areas. The EU proceeds on the assumption that a welfare maximum is more likely being achieved by market mechanisms than by monopolistic structures. Different countries implement this concept in different ways. It is often unclear, what kind of planning problems will evolve in the future und which individual market participant should plan what in the presence of deregulation measures.

For instance, the following scenarios are being discussed in bus traffic. Should a region specify the level of service in detail and only invite tenders for the operation of the trips, or should the regions evaluate offers of public transport companies, or should public transport companies act as providers of mobility in free competition? Air traffic has enjoyed an enormous boom because of the US-driven deregulation. Competition and the necessary, resulting optimization measures improved efficiency substantially. "Should wheel and track be split and what should be operated privately?" are controversial topics in the railway sector. The advocates of railway privatization refer to air traffic success stories, the opponents argue that an integrated system can be planned and controlled better, and is therfore more customer friendly.

A monopolistic approach offers a chance for an integrated system-wide optimization, while the creation of a market forces each participant to optimize his activities. No matter if and how regulation and deregulation take place, mathematics is always useful.

Regulation and deregulation, auctions and allocation mechanisms received a great deal of attention in the economic research community in recent years. One can, however, not by any means speak of a mathematical theory of regulation and deregulation yet. Up to now, quantitative implementations of theoretical concepts have only taken place in very special and individual areas such as auctioning truck-load contracts, mining rights, or telecommunication frequencies - not always to the satisfaction of all parties.

\subsection{Mathematical Models and Algorithms}

Discrete optimization is in general perfectly suited to formulate many planning problems in public transit, because they are often about yes/no decisions that can be modeled as $0 / 1$ optimization problems. Mathematical modelers have learned to express complex dependencies by means of linear equations and inequalities with integrality constraints and created, in this way, modeling tools that in principle allow to formulate problems in public transport at an arbitrary level of accuracy. This approach has several advantages. Traffic planning problems can be understood and described precisely, with all their constraints and prerequisites. In this way, discrete mathematics offers its services as a "language of traffic planning". It allows to communi- 
cate and analyze questions across companies and countries. Mathematical models help to structure problem areas, to recognize and point out special cases and generalizations. One should not conceal in this context that it is quite in-transparent for the layman whether a model is theoretically or practically easy or difficult to solve. Even experienced optimizers can often not tell a priori, whether and for which order of magnitude a concrete, difficult problem can be solved.

Standard Models and Techniques. Over time, a number of standard models of combinatorial optimization have proved to be useful in traffic applications (set partitioning, path covering, and network flows); these are complemented by general techniques such as the use of methods from linear and integer optimization. These methods and algorithms have been the subject of intensive work in the last decades. Today, problem sizes can be tackled that one would not have thought possible a few years ago. A study by Bob Bixby from 2004 showed that linear programs that took two months of computing time to solve in 1988, could be solved in a second in 2004. Faster computers contributed a speed-up factor of about 1600 to this, improvements in LP algorithms contributed a factor of about 3300 . Simply writing down mathematical models and applying standard techniques, however, is only sufficient to solve the problems of small PT companies, and for special applications. Often, minor-looking modifications, which are motivated by practical requirements, increase the level of difficulty significantly and must be faced by well-directed research efforts. The consideration of time windows, precedence constraints, path length restrictions etc. are typical examples for this.

Data for Academic Research. Applied algorithmic research on $\mathcal{N} \mathcal{P}$-hard problems can only succeed if practically relevant data is available, which can be used to test algorithmic alternatives experimentally. Availability of realworld data for academic research is of utmost value. One cannot avoid noticing that the situation in this area is very unsatisfactory. Even public transit companies that collect data with additional public funds, do (with very few exceptions) not even confidentially make this data available for academic research. Fear of leaking business secrets is a substantial obstacle to progress in the mathematical solution of planning problems in public transit.

Theory and Algorithms. Planning problems in the area of traffic and transport usually lead to combinatorial optimization problems with an extreme number of variables and/or constraints, which cannot be tackled by commercial linear or integer programming standard codes. In the last decade, 
techniques such as Lagrangean pricing or separation algorithms have been developed, which can deal with such problem sizes by means of dynamically generating the currently relevant variables and constraints on the fly, instead of dealing with every detail of the problem right from the beginning. These techniques allow to solve classical problems in traffic planning with sufficient quality.

These successes pave the way to the present research on a number of advanced topics:

- Coupling of discrete models (integrated planning). Up to now, success in the solution of coupled discrete models (e.g., vehicle and crew scheduling) has been limited to highly structured individual cases of limited size. The long-term goal is to overcome the artificial decomposition of the planning process into a hierarchical sequence of individual problems in order to produce an overall plan in one step, using a toolbox of models and methods that can be combined with each other. One approach to get the coupling of models to work uses methods of non-linear and non-differentiable optimization (sub-gradient and bundle methods). Substantial research and development efforts are still needed here.

- Robust and stochastic planning. The successes in classical optimization brought another aspect more and more into the focus of attention. It is that sophisticated plans are particularly vulnerable to disruptions. The overall goal must therefore be to compute plans that do not only look good on paper, but also turn out to be good in practice. Here, it is necessary to implement buffers in such a way that small disruptions can be intercepted without major operational changes. There are at present several, at least theoretically, competing approaches to deal with this question, in particular, methods of robust and stochastic optimization seem to be suitable. Practical applications and experiences, however, are virtually non-existent.

- Real-time planning. Traffic systems sometimes get out of hand because one cannot take all potential disruptions into account. Exactly these disruptions annoy passengers the most, in particular, if no information on the further course of the journey is available. Real-time optimization tries to tackle this kind of problems. One must admit, however, that the theoretical tools are currently not particularly advanced and that, specifically, hardly any useful statements about the practical performance of online algorithms can be made. It is therefore an important matter to develop theoretically well-founded methods for the real-time optimization of transportation systems that work in practice.

- Coupling of discrete, stochastic, and non-linear models (infrastructure 
planning). An area that is, from our point of view, nearly devoid of mathematics is the construction of the infrastructure of transportation systems. If anywhere, traditional planning methods are dominating the scene here. Decisions are made in a political way, considering some technical details, past experiences, and, in good cases, the use of simulation software.

The development of mathematical methods of infrastructure design is undoubtedly difficult, because it requires a combination of models from different mathematical areas, that employ, in particular, different mathematical tools. User behavior can only be predicted and must therefore modeled in a stochastic way, quantities such as revenue $=$ price $\times$ demand lead to non-linearities, monthly tickets to staircase functions and hence to discontinuities, the consideration of the tradeoff between quality and price to multi-criteria optimization, and this list can be continued arbitrarily.

Considering the long-term impact of these decisions and the pure investment volume, however, it would seem appropriate to undertake serious attempts towards a model-based long-term planning. The research community is investigating some of the relevant basic problems such as non-linear network flows, integer programs with certain non-linearities, or the general acceleration of stochastic optimization approaches by means of, e.g., scenario reduction, algorithmic game theory etc. First successes were scored in the application of discrete choice models to fare planning in public transit or in airline revenue management.

In the end, decisions about infrastructure construction will clearly not be made on the basis of a single mathematical model. Alternative computations and complex scenario simulations must also be considered as decision criteria.

\subsection{Transfer and Education}

Communication and Education. An obstacle to the exploitation of synergies between practitioners, algorithms designers, and theoreticians is a common lack of willingness to approach each other. Mathematicians do not want to enter the "lower depths" of data acquisition, while practitioners find the considerations of the mathematicians too far from reality. This division starts already in the language. A practitioner who talks about optimization is often thinking of a different thing than a mathematician. This communication problem is reinforced by ignorance of the contributions that the respective other group could make. What hits the eye in the context of 
this article is, in particular, a striking deficit in the education of traffic engineers with respect to modern methods of optimization, graph theory, and discrete mathematics. Every engineer knows what a differential equation is, but many have not learned that decision problems can be formulated and solved as integer programs, albeit this is the modern approach to good traffic planning. This is (all over the world) a clear shortcoming in university education, that must be remedied.

Business Environment. With the advent of mathematical planning in the airline industry, many companies set up operations research (or similarly termed) departments, which investigated the planning problems mentioned in this article and developed proprietary solutions. Over the years, most of these departments have been sourced out. The best among them became software companies that offer standardized solutions which are used by many airlines. In bus and rail traffic, on the other hand, such OR departments have not evolved to the same extent. Software for bus and railway companies is developed by suppliers with a university background. All these companies are small in comparison to their customers and have problems to impose their ideas and internal standards against the manifold of special requests of their big customers. These special requests fragment the software market and produce high costs, because they require cost intensive individual developments and maintenance. Small software companies also have problems to make enough advertisements to promote their innovations successfully.

\subsection{Conclusion}

Problems of infrastructure design, crew scheduling, etc. are for more than 100 years topical subjects of traffic engineering. They were always solved using the available technical and mathematical tools of the time. The dean of the traffic engineering faculty of the university of Dresden wrote in his obituary to the eminent traffic engineer Rüger:

"Using scientific insights to improve the work of public transit companies, their application and implementation in planning and operations, were always his maxim." (translation by the authors).

Rüger [31] in East Germany and Lehner [24] in West Germany wrote pioneering works on traffic planning, which describe, in principle, a mathematical model of a public transit company. At that time, the decision problems had to be reduced to (from today's point of view) simple, practically adequate, formulas, which constitute, even today, reasonable decision rules for practice. The progress in mathematics, however, now makes entirely new approaches possible, which derive decisions as solutions of complex network models. The 
goal must be to consequently develop these mathematics further, in order to continously improve the planning process in the sense of Rüger and Lehner, such that resources are saved, the use of public funds is reduced, and the customers enjoy a user friendly and adequate level of transportation services.

We see the transition from formula- to model-based mathematical planning (as we have sketched it) as a leap similar to the transition from the Braun tube to the transistor, or from the drawing board to the CAD system, and we are convinced that mathematics will more and more become a substantial production factor in public transit.

Despite the mentioned shortcomings in inter-disciplinary communication, in education, and in the transfer to practice, Germany has, in our opinion, excellent premises to help mathematical planning methods achieve a breakthrough in public transit. German traffic systems are among the best of the world, German software companies have leading positions in the world market, and German universities feature a variety of departments where scientists work on the quantitative treatment of planning problems in public transit on the highest international level. Public transit offers substantial optimization potentials. The know-how to exploit them is available. If we succeed in getting the different parties to cooperate in a coordinated and well-directed way, Germany could become a showcase of efficient public transit.

\section{$5 \quad$ Visions and Recommendations}

Our vision is to establish discrete mathematics and optimization as an essential production factor in all areas of traffic planning. The mathematical models and methods have to be built up such that they can support the various planning processes in practice in an adequate and user friendly way. This requires mathematical progress (in some areas definitely significant progress), but also the interfaces to the involved engineers and practitioners must be improved in a joint endeavor.

As we have shown in the preceeding sections, there is need for action in nearly all areas of public transportation planning. It reaches from mathematical modeling and the associated theory, via the implementation of this theory within algorithms, to the introduction of optimization systems in practice. This is an agenda for decades, which requires not only the overcoming of mathematical and company internal obstacles, but also the consideration of political conditions.

We want to propose here two concrete measures, which can be initiated on relatively short notice and which can be funded by the German ministries of education and research resp. industry and commerce (BMBF/BMWi) or 
the German science foundation (DFG) together with the industry. Both measures are characterized by the inter-disciplinarity that is typical for the traffic area. They combine mathematical research with engineering knowhow, business management, practical experiences, and the innovative use of IT systems.

\subsection{Discrete optimal control: real-time re-planning of traffic systems in case of disruptions}

Operational disruptions of traffic systems are virtually unavoidable. Larger disruptions require adaptations of the schedule, which must be initiated immediately after the incident. From a mathematical point of view, this is a topic of online or real-time optimization. The application of online optimization methods needs many premises. All data about the planned and the actual state of the traffic system must be available. In addition, forecasts about possible evolutions of the system and existing alternatives of action are needed. The goal is to bring the traffic system back into a state in which plan and status quo coincide to the largest possible extent and in which the "damage" is minimal.

Online planning is done today in the control centers of public transport companies by experienced dispatchers, who are supported by control systems and their visualization tools. The dispatching itself, however, is mainly done "by hand". In most cases, decision tables and rule sets are used. These give clear instructions, but do not provide for optimization. Online optimization is supposed to advance the pursuit of goals instead of the execution of rules. A change to this type of mathematical planning requires, in particular, adaptations of the IT infrastructure and the linkage of different databases, a non-trivial endeavor in information technology.

A fundamental problem in online optimization is the recording of data in order to analyze and reconstruct the sequence of events. Ideally, such data should not only record the executed operation, but also the causes for disruptions and the countermeasures that have been taken. Such data is usually not available. A research program in online optimization should already start at this level and establish a high-quality database.

It must be possible to use this data in simulation systems, such that exemplary verifications of individual scenarios are possible. As long as there is no practically proven online theory, it is necessary to use simulation tools in order to reconsider solution approaches. Simulation has a long tradition in the railway industry, where a number of systems such as RailSys, OpenTrack, etc. allow to produce an accurate picture of the real operation. In bus and air traffic, such simulation systems are still missing or under development. 
The real-time algorithms to be developed should take the dispatchers' years of experience and their heuristic scheduling rules into account. Cooperation between mathematicians, computer scientists, and experienced dispatchers is necessary. A possible additional progress that we see is that mathematical algorithms can monitor the state of a traffic system in the sense of a control circuit, in order to take preventative measures when changes in the state of the system are forecast or become apparent that can impair the operation. Ideas of this type could be summarized under the term "discrete optimal control". It refers to the continuous use of optimization algorithms to keep traffic systems on schedule. Similar questions come up, e.g., in mechanics and in production engineering. Their mathematical study is the subject of control theory, which typically uses methods from the theory of differential equations. The novelty in this case is that methods from discrete optimization play a prominent role as well.

Projects of this type could have been started 10 years ago. At that time, however, the IT base did not exist. Today we see a chance to bring know-how in information technology, mathematical methods, and operational experience together in a way that is beneficial for all participants. Such a project can range from the online optimization of subways, street cars, and commuter trains, via the real-time optimization of bus networks, to the real-time optimization of the entire German railway network or the entire European airspace; the last two of these applications are clearly still a long way off. From the customer's point of view it is important to combine online optimization and passenger information, such that there is an immediate benefit for the passengers.

\subsection{Model integration: service design in bus and rail traffic}

We have talked about various aspects of model integration and want to propose here the integration of mathematical models in the special area of service design in bus and rail transport. The goal is that the resulting models are algorithmically tractable and produce reasonable results from a practical point of view.

Similar as in the preceeding case of discrete optimal control, the data that is actually needed is not available at a sufficient level of accuracy.

The special characteristic of service design is a tight correlation of several aspects. We have already pointed out that demand forecasting is a stochastic problem, while network design leads to discrete optimization problems, and the determination of fares to non-linear mixed-integer optimization models. The integration of models from different mathematical disciplines is therefore a prerequisite for the development of successful approaches to service 
design. Model integration is in general hopelessly difficult, however, the special structures of this concrete application seem to present a chance for the successful development of adequate solution approaches.

First steps in this direction have been taken in toll collection applications using so-called bi-level optimization techniques. Non-linear optimization models and stochastic demand models (discrete choice Logit models) have been combined in the area of fare planning.

The procedure would be based upon traditional planning structures. Beyond the computation of a few scenarios, however, integrated models could take feedback and substitution effects into account, e.g., by explicitly including individual traffic (i.e., car traffic) in the models.

\section{References}

[1] R. E. Anbil, B. Patty Gelman, and R. Tanga. Recent advances in crewpairing optimization at American Airlines. Interfaces, 21:62-74, 1991.

[2] Cynthia Barnhart, Amy M. Cohn, Ellis L. Johnson, Diego Klabjan George L. Nemhauser, and Pamela H. Vance. Airline crew scheduling. In Randolph W. Hall, editor, Handbook of Transportation Science, pages 517-560. Kluwer, Boston, 1999.

[3] Cynthia Barnhart, Ellis L. Johnson, George L. Nemhauser, Martin W. P. Savelsbergh, and Pamela H. Vance. Branch-and-price: Column generation for solving huge integer programs. Operations Research, 46(3):316-329, 31998.

[4] Berliner Verkehrsbetriebe AöR (BVG). Business report 2006. http:// www.bvg.de/index.php/de/Common/Document/field/file/id/1409, 2006 .

[5] Andreas Bley and Marcus Pattloch. Modellierung und Optimierung der X-Win Plattform. DFN-Mitteilungen, 67:4-7, 2005.

[6] Ralf Borndörfer, Martin Grötschel, and Andreas Löbel. Duty scheduling in public transit. In Willi Jäger and Hans-Joachim Krebs, editors, MATHEMATICS - Key Technology for the Future, pages 653-674. Springer Verlag, Berlin, 2003.

[7] Ralf Borndörfer, Martin Grötschel, and Marc E. Pfetsch. A columngeneration approach to line planning in public transport. Transportation Science, 41(1):123-132, 2007.

[8] Sven Brieger. Workflow oriented and integrated optimization. Talk at the AGIFORS conference, May 2005. 
[9] Thomas M. Cook. Sabre soars. ORMS Today, 25(3):27-31, 61998.

[10] J. R. Daduna and A. Wren, editors. Computer-Aided Transit Scheduling, Lecture Notes in Economics and Mathematical Systems. Springer Verlag, Berlin, 1988.

[11] Joachim R. Daduna, Isabel Branco, and José M. Pinto Paixão, editors. Proc. of the Sixth International Workshop on Computer-Aided Scheduling of Public Transport (CASPT), Lisbon, Portugal, 1993, volume 430 of Lecture Notes in Economics and Mathematical Systems, Berlin, Heidelberg, 1995. Springer Verlag, Berlin.

[12] Camil Demetrescu, Andrew Goldberg, and David Johnson, editors. 9th DIMACS Implementation Challenge - Shortest Path, Piscataway, New Jersey, 2006. DIMACS.

[13] Guy Desaulniers and Jaques Desrosiers Marius M. Solomon, editors. Column Generation (Gerad 25th Anniversary). Springer Verlag, Berlin, 2005.

[14] M. Desrochers and J.-M. Rousseau, editors. Computer-Aided Transit Scheduling, Lecture Notes in Economics and Mathematical Systems. Springer Verlag, Berlin, 1992.

[15] Deutsche Bahn AG. Business report 2006. http://www.db.de/ site/bahn/de/unternehmen/investor_relations/finanzberichte/ geschaeftsbericht/geschaeftsbericht_2006.html, 2006.

[16] Deutsche Lufthansa AG. Business report 2006. http://konzern. lufthansa.com/de/downloads/presse/downloads/publikationen/ lh_gb_2006.pdf, 2006.

[17] Edsger Wybe Dijkstra. A note on two problems in connection with graphs. Numerische Mathematik, 1:269-271, 1959.

[18] Forschungsgesellschaft für Strassen- und Verkehrswesen. Heureka '05: Optimierung in Verkehr und Transport, Cologne, 2005.

[19] Michael R. Garey and David S. Johnson. Computers and intractability. Freeman, 1979.

[20] Martin Grötschel, Sven O. Krumke, Jörg Rambau, and Luis M. Torres. Making the yellow angels fly: Online dispatching of service vehicles in real time. SIAM News, 35(4):10-11, 2002.

[21] Karla L. Hoffman and Manfred Padberg. Solving airline crew scheduling problems by branch-and-cut. Management Science, 39(6):657-682, 1993. 
[22] Diego Klabjan, Ellis L. Johnson, George L. Nemhauser, Eric Gelman, and Srini Ramaswamy. Solving large airline crew scheduling problems: Random pairing generation and strong branching. Computational Optimization and Applications, 20(1):73-91, 2001.

[23] John O. Ledyard, Mark Olson, David Porter, Joseph A. Swanson, and David P. Torma. The first use of a combined-value auction for transportation services. Interfaces, 32(5):4-12, 2002.

[24] Friedrich Lehner. Der maximale Wirkungsgrad des Personaleinsatzes. Alba Verlag, Düsseldorf, 1978.

[25] Christian Liebchen. Periodic Timetable Optimization in Public Transport. Ph.D. thesis, TU Berlin, 2006.

[26] K. Littlewood. Forecasting and control of passenger bookings. In AGIFORS Symposium Proceedings, volume 12, Nathanya, Israel, 1972.

[27] Andreas Löbel. Optimal Vehicle Scheduling in Public Transit. Shaker Verlag, Aachen, 1997. Ph.D. thesis, TU Berlin.

[28] Martin Müller-Elscher. Die MICROBUS-Optimierungskomponenten im Überblick, talk at 1. PSYRUS user forum, 30. 6. 2005, Fulda.

[29] Marika Neumann. Mathematische Preisplanung im ÖPNV. Master's thesis, TU Berlin, 2005.

[30] Partner für Berlin Gesellschaft für Hauptstadt-Marketing GmbH. Berlinbrief, 2003. ISSN 1611-3284.

[31] Siegfried Rüger. Transporttechnologie städtischer öffentlicher Personenverkehr. Transpress, Berlin, 1986.

[32] Barry C. Smith, John F. Leimkuhler, and Ross M. Darrow. Yield management at American Airlines. Interfaces, 22(1):8-31, 1992.

[33] Andreas Sturmowski. Notwendigkeit der Effizienzsteigerung unter Einsatz von IT-Tools. In Proceedings der IVU-Konferenz IT im ÖPNV am 05.10.200\%. IVU Traffic Technologies AG, Berlin, 92007.

[34] Kalyan T. Talluri and Garrett J. van Ryzin. The Theory and Practice of Revenue Management, volume 68 of International Series in Operations Research and Management Science. Kluwer, Boston, MA, 2005.

[35] Stefan Voss and Joachim Daduna, editors. Computer-Aided Scheduling of Public Transport, Lecture Notes in Economics and Mathematical Systems. Springer Verlag, Berlin, 2001. 
[36] N. H. M. Wilson, editor. Computer-Aided Transit Scheduling, Lecture Notes in Economics and Mathematical Systems. Springer Verlag, Berlin, 1999.

[37] Gang Yu, editor. Operations Research in the Airline Industry. Kluwer, Dordrecht, 1998.

[38] Gang Yu, Michael Argüello, Gao Song, Sandra M. McCowan, and Anna White. A new era for crew recovery at Continental Airlines. Interfaces, 33(1):5-22, 2003. 